\title{
DHA/AA alleviates LPS-induced Kupffer cells pyroptosis via GPR120 interaction with NLRP3 to inhibit inflammasome complexes assembly
}

\author{
Guoqiang Fan', Yanfei Li', Jinglong Chen ${ }^{1}$, Yibo Zong ${ }^{1}$ and Xiaojing Yang (iD
}

\begin{abstract}
Pyroptosis is a novel type of programmed cell death associated with the pathogenesis of many inflammatory diseases. Docosahexaenoic acid (DHA) and Arachidonic acid (AA) is widely involved in inflammatory pathological processes. However, the effect and mechanism of DHA and AA on pyroptosis in Kupffer cells are poorly understood. The present study demonstrated that DHA and AA ameliorated lipopolysaccharide (LPS)-induced Kupffer cells pyroptosis by reversing the increased expression of NLRP3 inflammasome complex, GSDMD, IL-1ß, IL-18, and PI-stained positive rate. Next, the study revealed that GPR120 silencing eliminated the anti-pyroptosis of DHA and AA in LPS-induced Kupffer cells, suggesting that DHA and AA exerted their effect through GPR120 signaling. Importantly, GPR120 endocytose and binds to NLRP3 under LPS stimulation. Furthermore, co-immunoprecipitation showed that DHA and AA promoted the interaction between GPR120 and NLRP3 in LPS-exposed Kupffer cells, thus inhibiting the self-assembly of NLRP3 inflammasome complex. Finally, the study verified that DHA and AA alleviated hepatic injury through inhibiting Kupffer cells pyroptosis in vivo. The findings indicated that DHA and AA alleviated LPS-induced Kupffer cells pyroptosis via GPR120 interaction with NLRP3, it might become a potential therapeutic approach hepatic injury.
\end{abstract}

\section{Introduction}

The liver is the largest organ in the body responsible for nutrients metabolism. Hepatic injury is perceived as one of the major problems threatening human health; it is often associated with increased hepatic exposure to lipopolysaccharide (LPS). In the microenvironment of the liver, Kupffer cells are the first barrier defending against pathogens by sensing damage stimulus and producing various cytokines ${ }^{1,2}$. The innate-immune-dominated tissue inflammatory response initiated by Kupffer cells after hepatic injury involves many regulatory mechanisms ${ }^{3,4}$.

Pyroptosis is a novel type of programmed cell death discovered and proved in recent years. It is initiated by the activation of NOD-like receptor pyrin domain-containing protein 3 (NLRP3) inflammasome and performed by

Correspondence: Xiaojing Yang (yangxi@njau.edu.cn)

${ }^{1} \mathrm{MOE}$ Joint International Research Laboratory of Animal Health and Food Safety, Nanjing Agricultural University, Nanjing 210095, P. R. China

Edited by B. Zhivotovsky gasdermin D (GSDMD) protein. As a result, the activated GSDMD constitutes nanopores in the cell membrane, resulting in cell swelling, cell membrane rupture, and even cell death ${ }^{5,6}$. NLRP3 inflammasome is a multi-protein complex comprising a sensor NLRP3, an adaptor molecule apoptosis-associated speck-like protein containing a caspase recruitment domain CARD (ASC), and an effector caspase- $1^{7}$. Pyroptosis is involved in many liver diseases, such as hepatocytic injury and inflammation ${ }^{8}$. Therefore, the inhibition of NLRP3 inflammasomeinitiated pyroptosis is a new strategy for the preventing and treating LPS-induced liver injury.

Polyunsaturated fatty acids (PUFAs) are important nutrients in the human body involved in physiological and pathological processes ${ }^{9,10}$. Docosahexaenoic acid (DHA) is the most important one of the omega-3 PUFAs ${ }^{11}$. Previous studies demonstrated that DHA inhibited inflammation and had beneficial effects on various inflammatory diseases ${ }^{11-14}$. Arachidonic acid (AA) is a representative type

\section{(c) The Author(s) 2021}

(c) (i) Open Access This article is licensed under a Creative Commons Attribution 4.0 International License, which permits use, sharing, adaptation, distribution and reproduction cc) in any medium or format, as long as you give appropriate credit to the original author(s) and the source, provide a link to the Creative Commons license, and indicate if changes were made. The images or other third party material in this article are included in the article's Creative Commons license, unless indicated otherwise in a credit line to the material. If material is not included in the article's Creative Commons license and your intended use is not permitted by statutory regulation or exceeds the permitted use, you will need to obtain permission directly from the copyright holder. To view a copy of this license, visit http://creativecommons.org/licenses/by/4.0/. 
of omega-6 PUFAs and increasing dietary intake of omega6 fatty acids increases inflammation ${ }^{15}$. However, studies on healthy adults have found that an increased intake of AA did not increase the concentrations of inflammatory cytokines $^{16}$. Therefore, the role of AA in the inflammatory environment is complex and has not yet been properly understood. Furthermore, whether DHA and AA exert effects on pyroptosis in LPS-induced Kupffer cells has not been reported. G protein-coupled receptor 120 (GPR120, also known as FFAR4: free fatty acid receptor 4) is the receptor for PUFAs ${ }^{17-19}$ and has been located in adipocytes $^{20}$, macrophages ${ }^{18}$, and Kupffer cells ${ }^{21}$. DHA regulated anti-inflammatory and insulin-sensitizing effects through GPR120 signaling ${ }^{17,18,22,23}$. Agonist activation causes the redistribution of GPR120 away from the cell surface into internal cellular compartments through endocytosis known as internalization. Previous studies reported that DHA and AA were associated with marked GPR120 internalization ${ }^{19,24}$. However, whether GPR120 mediates DHA and AA action in pyroptosis and how it works have not been reported.

This study was novel in reporting that DHA/AA alleviated Kupffer cells pyroptosis via GPR120 interaction with NLRP3 in LPS-treated Kupffer cells. Thus, the study provided an in-depth resource and framework for understanding the effects of DHA/AA while suggesting approaches for hepatic injury therapy.

\section{Results}

\section{DHA/AA inhibited NLRP3 inflammasome-mediated} pyroptosis in LPS-induced Kupffer cells

Various concentrations $(0,10,50,100 \mu \mathrm{M})$ of DHA/AA treated for $4 \mathrm{~h}$ had no effect on cell viability (Supplementary Fig. 1A, B). NLPR3 inflammasome-mediated pyroptosis is a novel programmed cell death that involved in liver inflammation. Pyroptosis was assessed by detecting the level of pyroptosis key proteins to determine the influence of DHA/ AA on pyroptosis in LPS-induced Kupffer cells. Quantitative real-time PCR and western blot analysis showed that the expression of NLRP3, ASC, and caspase- 1 significantly increased in the LPS group compared with the Con group (Fig. 1A-F). Immunofluorescence analyses further confirmed that the expression of NLRP3 strongly increased in the LPS group compared with the Con group (Fig. 1G). However, the LPS-induced effect was significantly reversed by DHA or AA supplementation.

In addition, quantitative real-time PCR and western blot analysis showed that the expression of GSDMD, interleukin-1 $\beta$ (IL-1 $\beta$ ), and IL-18 significantly increased in the LPS group compared with the Con group (Fig. 2A-G). Enzyme-linked immunosorbent assay (ELISA) analyses further showed that the secretion of IL-1 $\beta$ and IL-18 significantly increased in the LPS group compared with the Con group (Fig. 2H, I). However, LPS-stimulated expression of GSDMD, IL-1 $\beta$, and IL-18 was significantly reversed by DHA or AA treatment.

Kupffer cells pyroptosis was detected using scanning electron microscope (SEM). The holes in the cell membrane and the rupture of the cell surface were clearly visible in the LPS group compared with the Con group, while the pyroptosis phenotypes were improved by DHA or AA supplementation (Fig. 2J). Likewise, the result of propidium iodide (PI) staining was consistent with the change in cellular phenotype (Fig. 2K).

\section{DHA/AA alleviated inflammation response in LPS-induced Kupffer cells}

Kupffer cells were treated with DHA/AA at various concentrations and time course to evaluate their effect on inflammatory responses. The expression of IL- $1 \beta$ and IL-18 strongly decreased in the 50 and $100 \mu \mathrm{M}$ DHA/AA group compared with the LPS group (Supplementary Fig. 1C-F). In addition, the expression of IL-1 $\beta$ and IL-18 significantly decreased after treatment with $50 \mu \mathrm{M}$ DHA/AA for 4 or $12 \mathrm{~h}$ compared with the LPS group (Supplementary Fig. 1G-J).

Quantitative real-time PCR showed that the mRNA expression of IL-6, MCP-1, and iNOS significantly increased in the LPS treatment group compared with the Con group (Supplementary Fig. 2A-C). Western blot analysis showed that the protein expression of tumor necrosis factor $\alpha$ (TNF- $\alpha$ ) and cyclooxygenase-2 (COX2) significantly increased in the LPS group compared with the Con group (Supplementary Fig. 2D-F). However, the expression of IL- 6, MCP-1, iNOS, TNF- $\alpha$, and COX2 significantly decreased in the DHA or AA supplementation group compared with the LPS group (Supplementary Fig. 2A-F).

\section{Interference with GPR120 eliminated the effects of DHA/ AA on NLRP3 inflammasome-mediated Kupffer cells pyroptosis}

Previous studies demonstrated that DHA antiinflammatory activity in macrophages was GPR120dependent ${ }^{13,18}$. However, whether AA can activate GPR120 or whether DHA and AA can exert anti-pyroptosis effects through GPR120 was unclear. To address this, siGPR120 (siRNA1, siRNA2, and siRNA3) was transfected to achieve GPR120 silencing, and quantitative real-time PCR (Supplementary Fig. 3A) and western blot analysis were performed to verify the transfection efficiency (Supplementary Fig. 3B). Subsequently, GPR120 siRNA3 was chosen for subsequent experiments and the mRNA expression of GPR120 significantly decreased after using GPR120 siRNA compared with the NC siRNA group (Fig. 3A). Quantitative real-time PCR and western blot analysis showed that the expression of NLRP3, caspase-1, and GSDMD significantly increased in the LPS group compared with the Con group (Fig. 3B-H). ELISA analyses further showed that the 


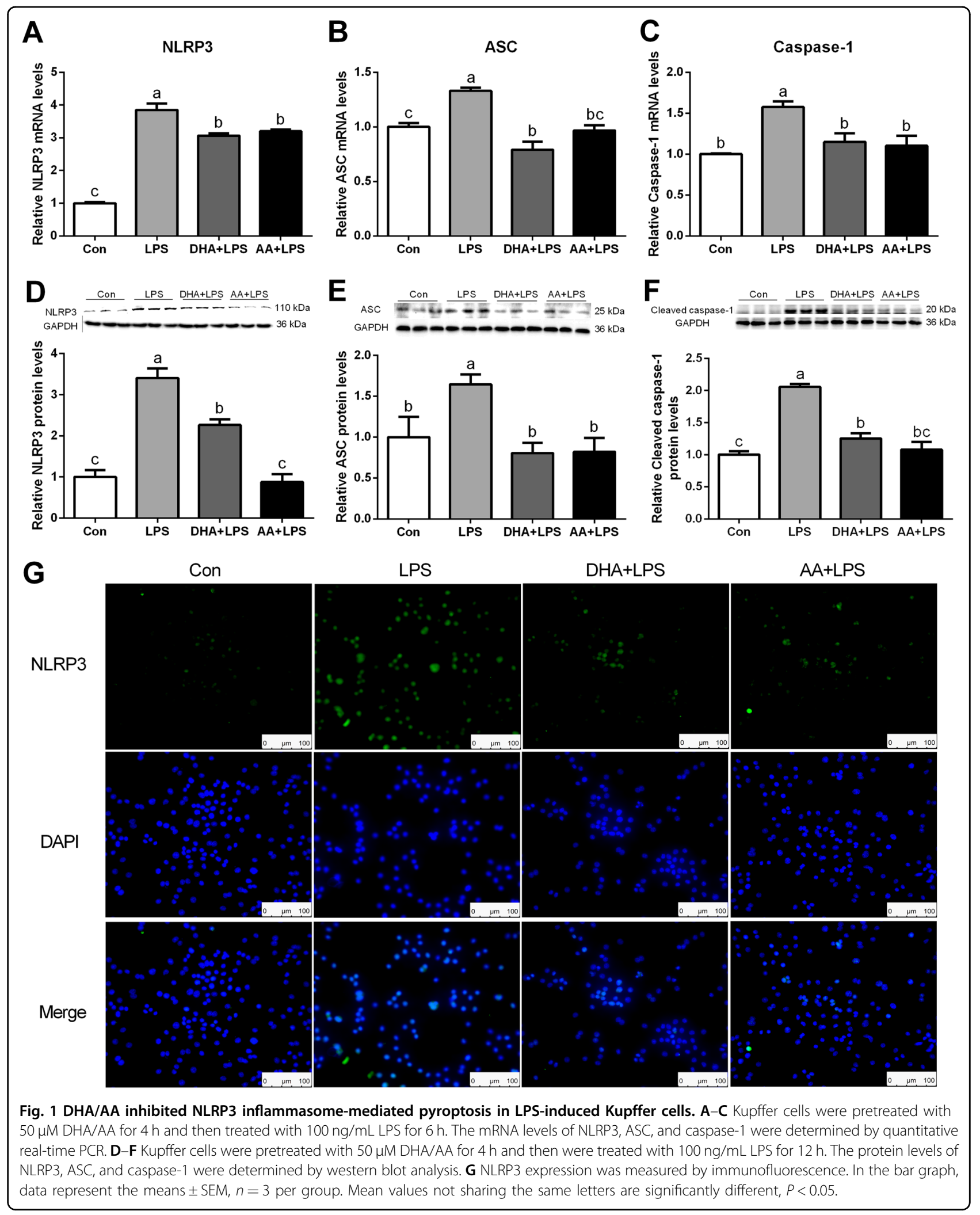




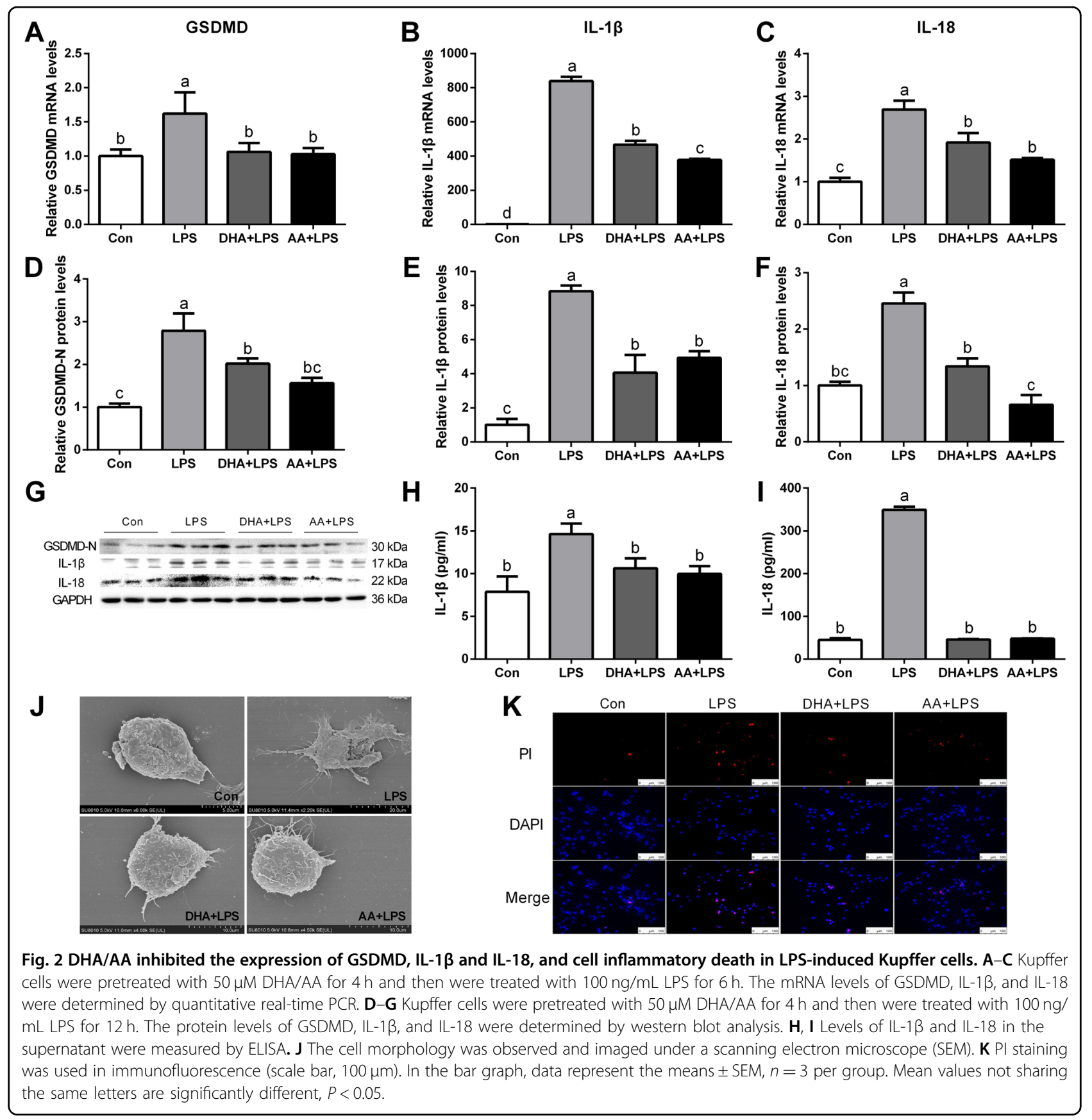

secretion of IL-1 $\beta$ and IL-18 significantly increased in the LPS group compared with the Con group (Fig. 3I, J). However, the LPS-induced effects were not improved by DHA or AA supplementation after GPR120 silencing. Also, PI staining revealed similar results (Fig. 3K).

DHA/AA promoted GPR120 interaction with NLRP3 and decreased NLRP3 inflammasome assembly in LPS-induced Kupffer cells

Kupffer cells were treated with LPS for $12 \mathrm{~h}$ to examine how GPR120 affected pyroptosis. The GPR120 protein expression significantly decreased in the plasma membrane after LPS treatment. In contrast, LPS stimulation significantly increased the protein expression of GPR120 in the cytoplasm (Fig. 4A). Then, the samples for the immunoprecipitation (IP) of GPR120 were used to detect the protein that interacted with GPR120 with the help of liquid chromatography with tandem mass spectrometry (LC-MS/MS). Venn diagram showed the detected protein in the Con and LPS groups; NLRP3 was observed in the LPS group (Fig. 4B). In addition, immunofluorescence revealed the co-localization of GPR120 and NLRP3 in 


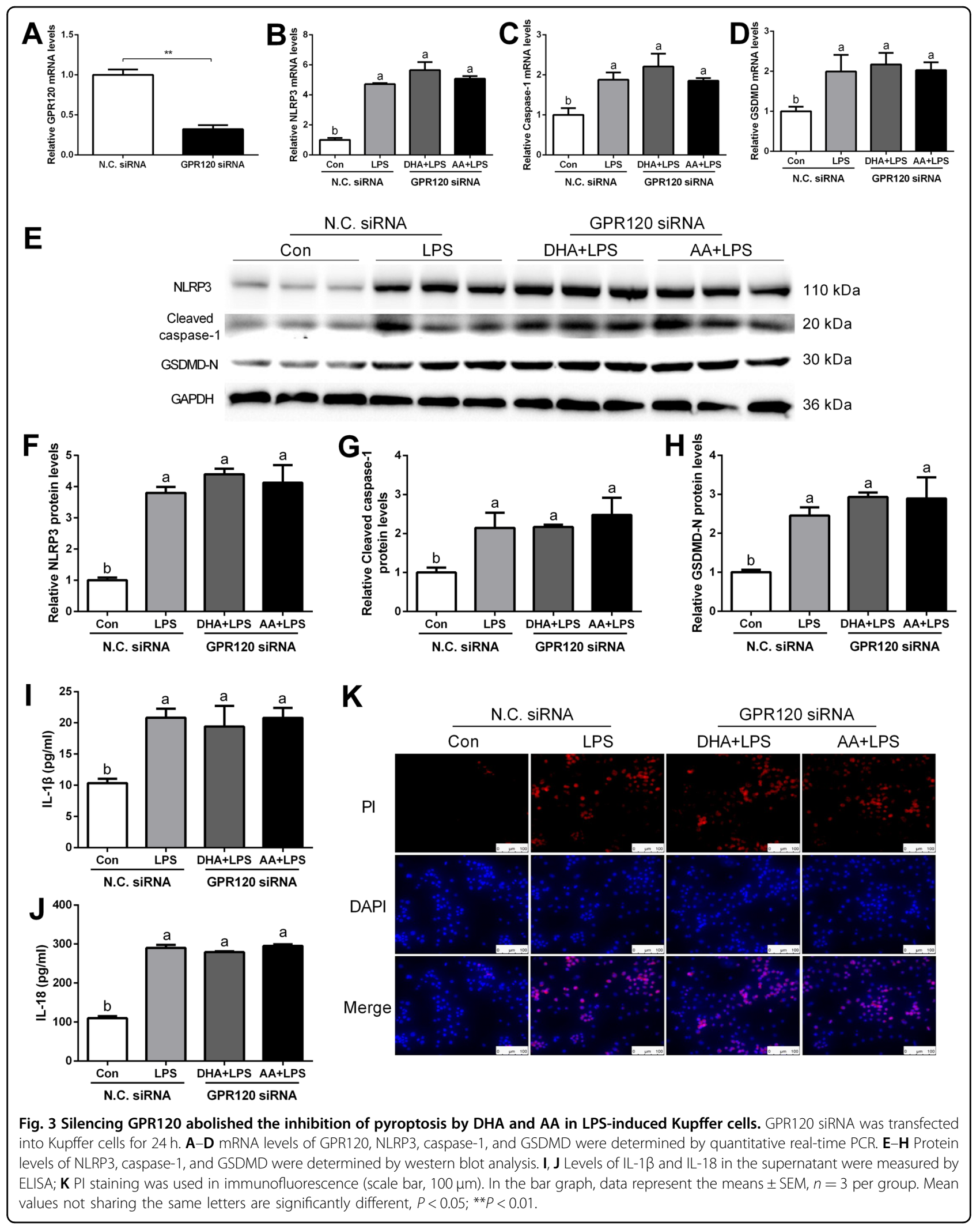



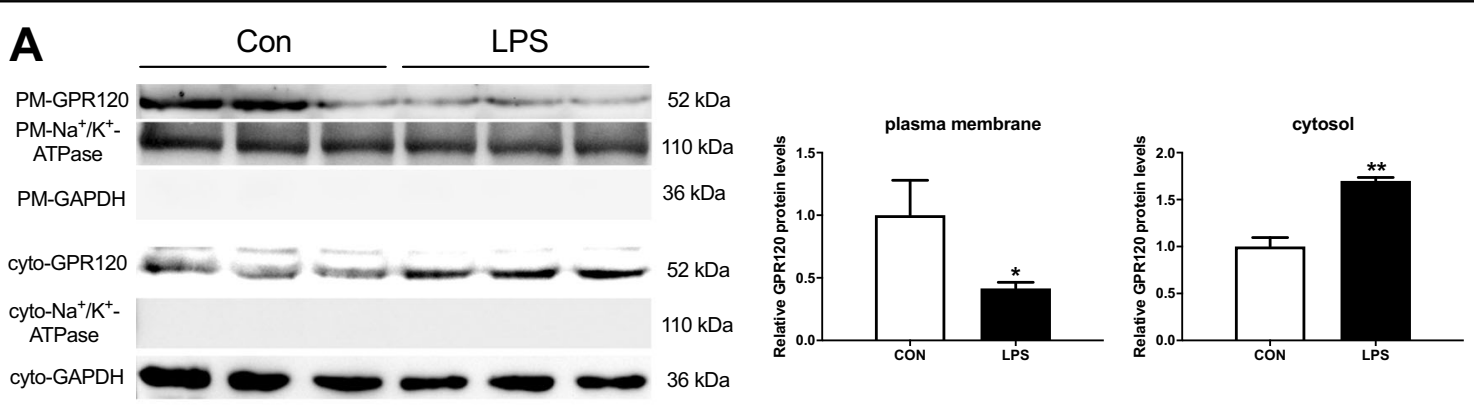

B
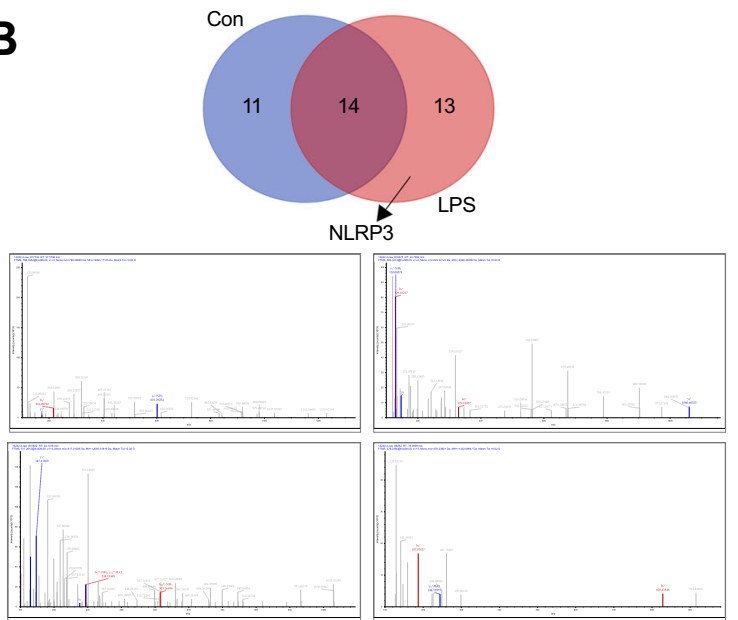

C

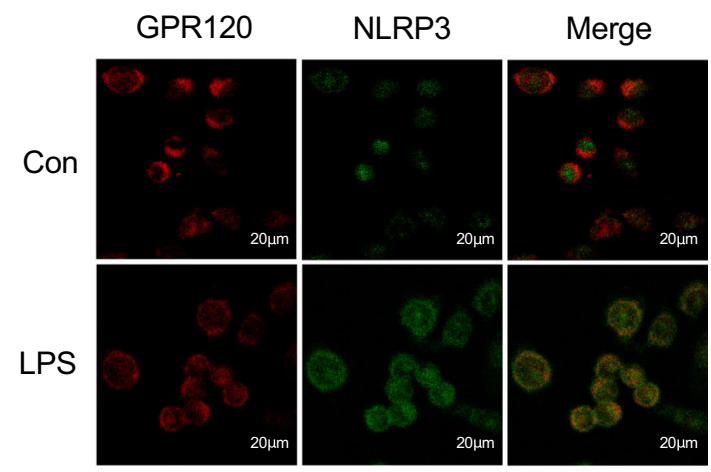

D

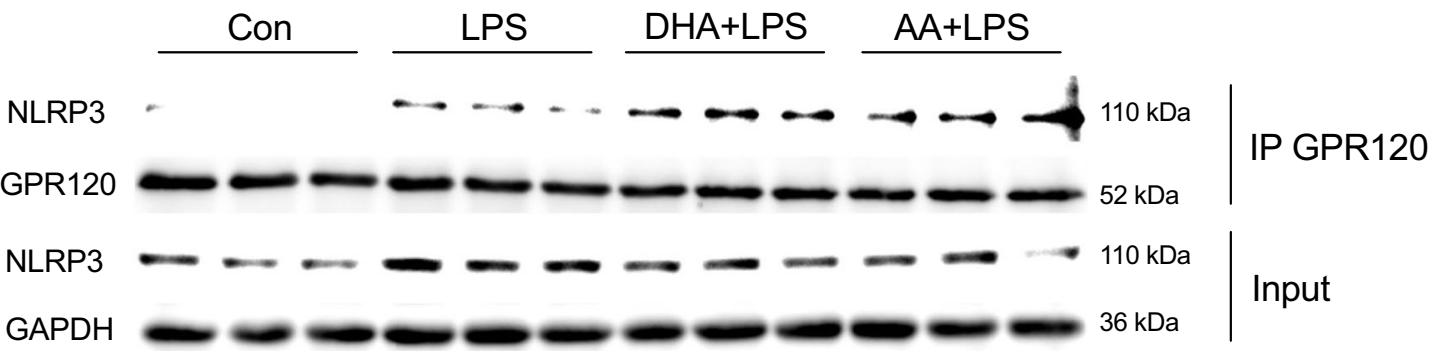

E

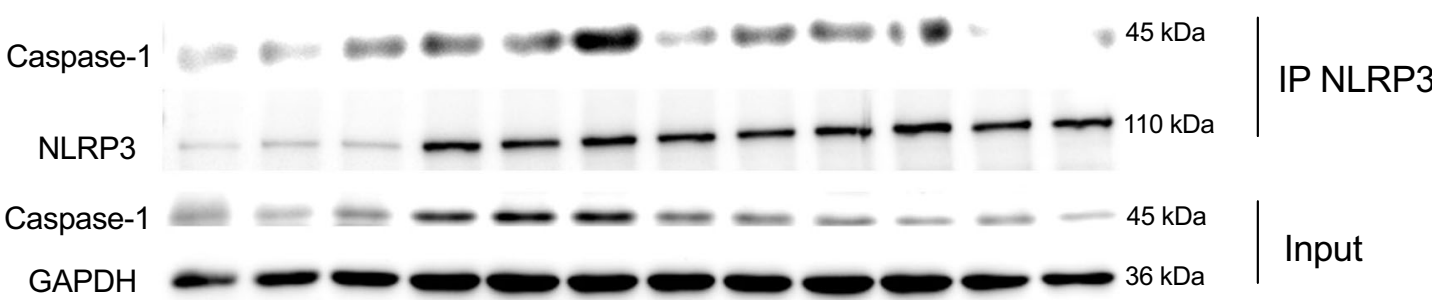

Fig. 4 DHA/AA promoted the interaction between GPR120 and NLRP3 in LPS-induced Kupffer cells. Kupffer cells were treated with $100 \mathrm{ng} / \mathrm{mL}$ LPS for $12 \mathrm{~h}$. A The GPR120 protein expression in the plasma membrane and cytoplasm. B The expression of NLRP3 protein was observed by the Venn diagram and LC-MS/MS analysis (the upper image is the Venn diagram and the lower one is the NLRP3 mass spectrogram). C GPR120 and NLRP3 co-localization was observed in immunofluorescence (scale bar, $20 \mu \mathrm{m}$ ); D, E Kupffer cells were pretreated with $50 \mu \mathrm{M}$ DHA/AA for $4 \mathrm{~h}$ and then treated with $100 \mathrm{ng} / \mathrm{mL}$ LPS for $12 \mathrm{~h}$. The cell lysates were immunoprecipitated with GPR120 antibody and NLRP3 antibody separately, and then the samples were analyzed by western blot for GPR120, NLRP3, and caspase-1 as indicated in the text. In the bar graph, data represent the means \pm SEM, $n=3$ per group. ${ }^{*} P<0.01$ and ${ }^{*} P<0.05$. 


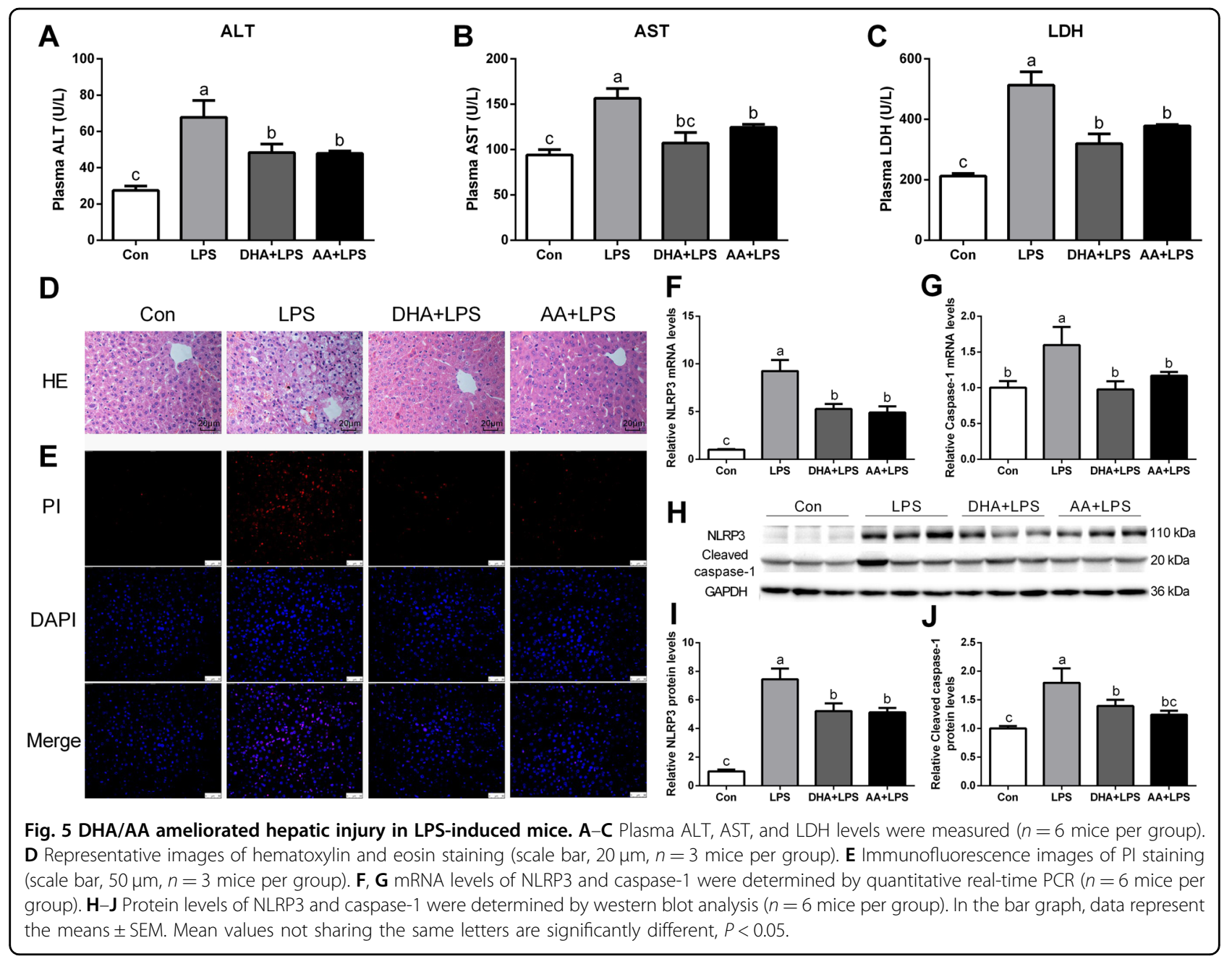

the LPS group (Fig. 4C). Next, co-IP was used to explore whether the interaction between GPR120 and NLRP3 contributed to the anti-pyroptosis effects of DHA/AA. The results shown in Fig. 4D indicated that DHA and AA promoted the interaction between GPR120 and NLRP3, thus decreasing the self-assembly of inflammasome complex. In addition, the decreased interaction between NLRP3 and caspase-1 was observed after DHA and AA supplementation (Fig. 4E). This finding further confirmed that DHA and AA via GPR120 interacted with NLRP3 and decreased NLRP3 inflammasome assembly to alleviate LPS-induced Kupffer cells pyroptosis.

\section{DHA/AA protected mice from LPS-induced hepatic injury}

Pretreatment with DHA and AA significantly improved LPS-induced liver injury as evidenced by reduced plasma alanine aminotransferase (ALT), aspartate transaminase (AST), and lactate dehydrogenase (LDH) levels (Fig. 5A-C). Also, hematoxylin and eosin (H\&E) staining showed that inflammatory cell infiltration was clearly visible in the LPS group compared with the Con group, while the hepatic injury phenotypes were improved by DHA or AA supplementation (Fig. 5D). Likewise, the result of PI staining was consistent with the change in the hepatic phenotype (Fig. 5E). Quantitative real-time PCR and western blot analysis showed that the expression of NLRP3 and caspase-1 significantly increased in the LPS group compared with the Con group (Fig. 5F-J). The LPSinduced effects were significantly ameliorated by DHA or AA supplementation.

\section{DHA/AA alleviated pyroptosis in LPS-induced primary Kupffer cells}

Primary Kupffer cells were isolated from the livers of LPS-exposed mice after pretreatment with DHA and AA. The purity of the Kupffer cells fraction was determined using the F4/80 antibody. The results of flow cytometry showed that F4/80-positive cells accounted for $85.44 \%$ of all cells (Fig. 6A). Quantitative real-time PCR and western blot analysis showed that the expression of NLRP3, caspase-1, and GSDMD significantly increased in the LPS group compared with the Con group (Fig. 6B-H). 


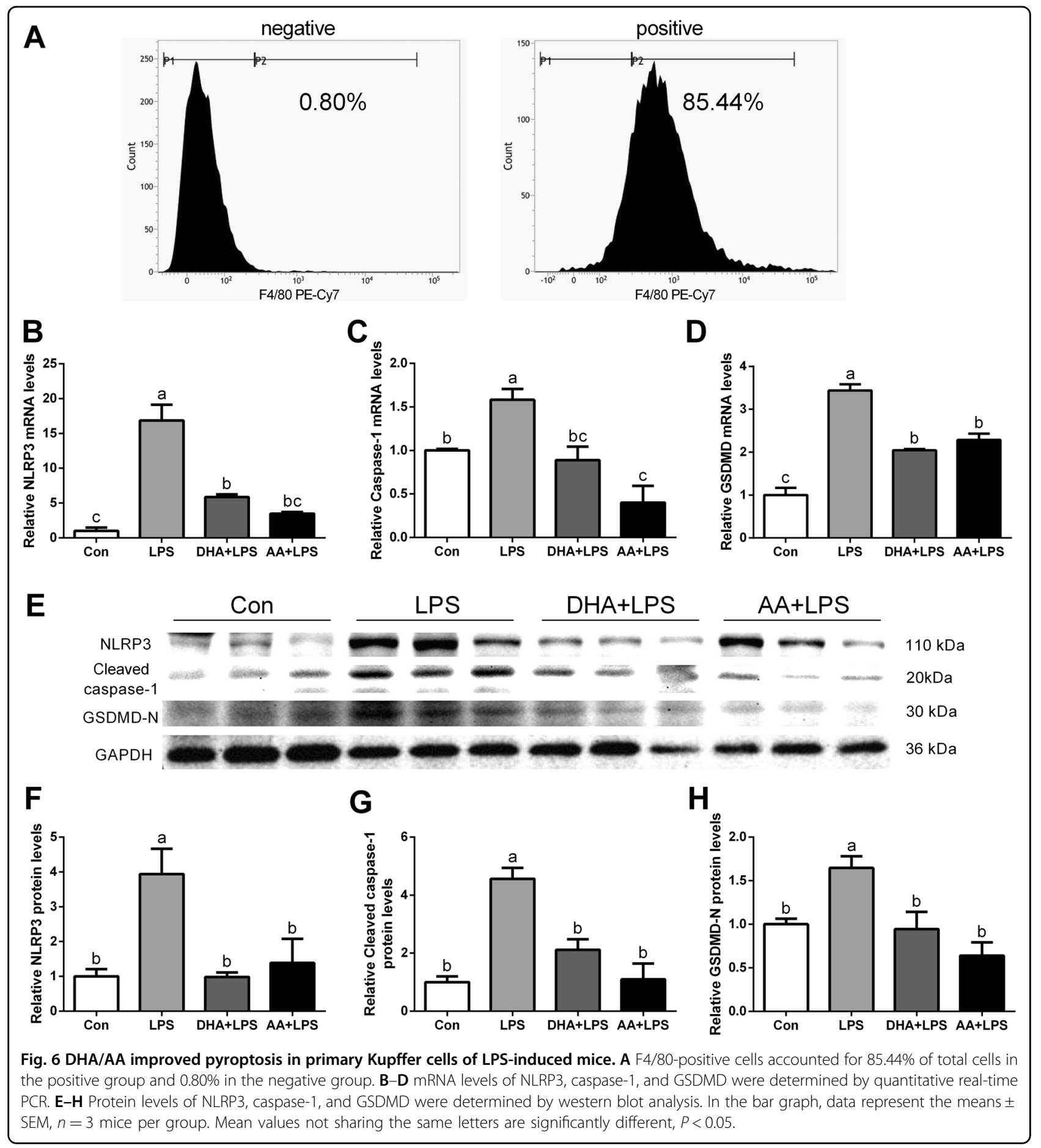

The expression of NLRP3, caspase-1, and GSDMD significantly decreased in the DHA or AA supplementation group compared with the LPS group (Fig. 6B-H).

\section{Discussion}

Kupffer cells, as the main macrophages in the liver, have been shown to exert significant effects in some liver diseases $^{25-27}$. This study showed that DHA and AA exhibited anti-pyroptosis activity in LPS-exposed Kupffer cells. The effects of DHA and AA were achieved through the interaction between GPR120 and NLRP3. Moreover, in vivo, DHA and AA supplementation successfully improved LPS-induced hepatic injury in mice by alleviating Kupffer cells pyroptosis.

Pyroptosis has been implicated in various diseases, such as inflammation, nonalcoholic fatty liver disease, and liver 
fibrosis $^{28-31}$. Bacteria, toxins and so on can elicit a strong immune response in macrophages, resulting in pyroptosis $^{29,32}$. However, the molecular basis of Kupffer cells pyroptosis is incompletely understood, which has limited the identification of therapeutic targets. DHA and AA are both long-chain PUFAs. DHA was found to suppress the progression of hepatic inflammation and inhibits hepatocyte pyroptosis induced by hepatic ischemia-reperfusion ${ }^{33}$. However, the effect of DHA on LPS-induced Kupffer cells pyroptosis and its mechanism are still unclear. In addition, the role of AA in the inflammatory environment is controversial and has not yet been properly understood. Thus, Kupffer cells were pretreated with DHA/AA and then treated with LPS to determine the influence of DHA/AA on pyroptosis in this study. Pyroptosis is inflammatory cell death mediated by NLRP3 inflammasome-dependent activation of caspase- $1^{34,35}$. The ASC participates in the NLRP3 inflammasome mediated activation of caspase- 1 and forms activated inflammasome complexes ${ }^{36}$. The activation of caspase-1 leads to the maturation of proinflammatory cytokines IL-1 $\beta$ and IL-18, which are released from the GSDMD pores on the plasma membranes and stimulate the immune cells to induce an inflammatory form of cell death known as pyroptosis ${ }^{37}$. This study was novel in reporting that DHA and AA alleviated the progression of pyroptosis in LPS-exposed Kupffer cells. In addition, pyroptosis is a kind of programmed cell death characterized by cell membrane rupture and perforation. This study showed that the pyroptosis phenotypes were improved by DHA or AA supplementation in LPS-exposed Kupffer cells.

GPR120 recognizes PUFAs ${ }^{17-19}$ and is expressed in Kupffer cells ${ }^{21}$, macrophages ${ }^{18}$, adipose tissue ${ }^{20}$, and intestinal $\operatorname{tract}^{17}$. Therefore, GPR120 siRNA was synthesized to transfect Kupffer cells so as to elucidate whether DHA and AA alleviated pyroptosis through GPR120 signaling. The results showed that GPR120 silencing tended to abolish these anti-pyroptosis actions of DHA and AA in LPSexposed Kupffer cells. These results suggested that DHA and AA alleviated pyroptosis of LPS-induced Kupffer cells via GPR120.

The study further investigated the mechanism underlying the interaction between GPR120 and pyroptosis. Previous studies reported that GPR120 mediated antiinflammatory effects via internalization ${ }^{18}$. First, the study revealed that a weaker GPR120 plasma membrane signal contrasted with a stronger GPR120 cytoplasmic signal in the LPS group compared with the Con group, suggesting that GPR120 was transferred from the plasma membrane to the cytoplasm to function after LPS treatment. The anti-inflammatory effects mediated by GPR 120 depended on its binding to downstream target molecules ${ }^{38,39}$. The study demonstrated GPR120 binding to NLRP3 using LCMS/MS and immunofluorescence after LPS treatment. Next, co-IP was used to explore whether the interaction between GPR120 and NLRP3 contributed to the antipyroptosis effects of DHA and AA. The results showed that DHA and AA promoted the interaction between GPR120 and NLRP3, thus inhibiting the self-assembly of the NLRP3 inflammasome complex. The formation of the NLRP3 inflammasome complex requires caspase-1 involvement ${ }^{40}$. This study found that the supplementation with DHA and AA reduced the binding of between NLRP3 and caspase-1. This finding further confirmed that DHA and AA via GPR120 interacted with NLRP3 and inhibited the NLRP3 inflammasome complex assembly to alleviate LPS-induced Kupffer cells pyroptosis.

In support of these findings, mice were pretreated with DHA and AA in vivo and then intraperitoneally injected with LPS to induce hepatic injury and pyroptosis. Previous studies reported that omega-3 PUFA prevented diet-induced nonalcoholic steatohepatitis ${ }^{13}$.The results showed that DHA and AA significantly improved LPSinduced liver injury. In addition, DHA and AA significantly inhibited the expression of NLRP3 and caspase1 in LPS-treated mouse liver. Previous studies showed that GSDMD-driven pyroptosis in immune cells aggravated hepatic ischemia-reperfusion injury ${ }^{41}$. Therefore, primary Kupffer cells were isolated from the livers of LPSexposed mice after pretreatment with DHA and AA. The study showed a significant decrease in primary Kupffer cells pyroptosis in LPS-exposed mice pretreated with DHA and AA, suggesting that DHA and AA might alleviate liver injury though inhibiting Kupffer cells pyroptosis.

Taken together, the data showed that DHA and AA alleviated LPS-induced Kupffer cells pyroptosis via GPR120 interaction with NLRP3 to inhibit NLRP3 inflammasome complex assembly. These findings provided new insights into the treatment of hepatic injury.

\section{Material and methods Regents}

DHA (HY-B2167) was purchased from MCE (MedChemExpress, New Jersey, USA) and AA (C4223) were purchased from APExBIO (Achieve Perfection, Explore the Unknown, Houston, USA). LPSs from Escherichia coli O55:B5 (LPS, L2880) and 4',6-diamidino-2-phenylindole (DAPI, D9542) were obtained from Sigma-Aldrich. NLRP3 (BS90949: Bioworld, diluted 1:100) and GPR120 (sc-390752: Santa Cruz, diluted 1:50) were used for immunofluorescence. PI (SL7090) was purchased from Coolaber Science \& Technology Co., Ltd (Beijing, China).

\section{Cell culture and cell transfection}

Kupffer cells (NASH-associated mouse Kupffer cell lines, BeNa Culture Collection, China, BNCC340733) were cultured in Roswell Park Memorial Institute 1640 medium (Cat No. 350-000-CL, Wisent, Nanjing, China) 
containing $10 \%(\mathrm{v} / \mathrm{v})$ fetal bovine serum at $37^{\circ} \mathrm{C}$ in an atmosphere of $5 \% \mathrm{CO}_{2}$. At $70 \%$ confluent growth Kupffer cells were pretreated with $50 \mu \mathrm{M}$ DHA/AA for $4 \mathrm{~h}$ and then treated with $100 \mathrm{ng} / \mathrm{mL}$ LPS for 6 or $12 \mathrm{~h}$.

For GPR120 knockdown, specific small interfering RNAs (GPR120 siRNA1, GPR120 siRNA2, and GPR120 siRNA3; GenePharma, Shanghai, China) were transfected into Kupffer cells using jetPRIME ${ }^{\circledR}$ transfection reagent (Polyplus Transfection, Beijing, China). The scramble siRNA was used as a negative control (NC siRNA). The sequences are listed in Supplementary Table S1.

\section{Animal experiment}

A total of 36 male specific-pathogen-free, aged 6 weeks, C57BL/6J mice were purchased from Yangzhou University Comparative Medical Center. All the mice were housed at $22 \pm 1{ }^{\circ} \mathrm{C}$, with a 12-h light/dark cycle and fed in the Animal Experiment Center, Nanjing Agricultural University. The mice were allowed to adapt to their environment for 1 week. After that, the mice were randomly assigned to four groups: the control (Con) group $(n=9)$, the LPS group $(n=9)$, DHA and LPS (DHA + LPS) group $(n=9)$, or AA and LPS (AA + LPS) group $(n=9)$. The mice in the DHA + LPS and AA + LPS groups were treated with $50 \mathrm{mg} / \mathrm{kg}$ DHA and AA, respectively. DHA and AA were dissolved in 5\% gum arabic solution and administered orally once daily via a gastric tube for 1 week before LPS treatment. The mice in the Con and LPS groups were gavaged with an equal volume of $5 \%$ gum arabic solution. During the treatment, all mice had free access to water and food. After 1 week of gavage, the mice in the LPS, DHA + LPS, and AA + LPS groups received an intraperitoneal injection of LPS at a dose of $5 \mathrm{mg} / \mathrm{kg}$, and the Con group mice received an intraperitoneal injection of an equal volume of physiological saline solution. After $12 \mathrm{~h}$, the livers from three mice were collected from each group for isolating Kupffer cells, and livers and blood from the other six mice were collected and stored in $-80{ }^{\circ} \mathrm{C}$ for later use.

This study was approved by the Animal Ethics Committee of Nanjing Agricultural University, China. Euthanasia and sampling procedures complied with the "Guidelines on Ethical Treatment of Experimental Animals" (2006) No. 398 published by the Ministry of Science and Technology, China, and with the "Regulation Regarding the Management and Treatment of Experimental Animals" (2008) No. 45, published by the Jiangsu Provincial People's Government.

\section{Primary Kupffer cells isolation and identification}

Primary Kupffer cells were isolated separately from C57BL/6 mice in the Con group, LPS group, DHA + LPS group, and AA + LPS group as previously described ${ }^{42}$.
Briefly, the liver was perfused with $10 \mathrm{~mL}$ of phosphatebuffered saline and then digested with $0.1 \%$ type IV collagenase. Following digestion, the liver homogenate was filtered through a $75-\mu \mathrm{m}$ stainless steel wire mesh to remove undigested tissue. The cell suspension was centrifuged at $50 \mathrm{~g}$ (Eppendorf $5810 \mathrm{R}$, Germany) for $5 \mathrm{~min}$ at $4{ }^{\circ} \mathrm{C}$. The top suspension was separated with $60 \%$ Percoll and then centrifuged at $2500 \mathrm{~g}$ for $25 \mathrm{~min}$. The darker layer in the middle comprised Kupffer cells. Kupffer cells were identified by flow cytometry using a monoclonal anti-F4/ 80 antibody (123114; Biolegend, California, USA) conjugated with $\mathrm{PE} / \mathrm{Cyanine7}$ (PE-Cy7). Flow cytometry was performed for detecting the percentage of F4/80-positive cells. Briefly, the collected cells were incubated with the antibody (1:100 dilution) for $30 \mathrm{~min}$ at $37^{\circ} \mathrm{C}$. The data were collected using a flow cytometer.

\section{CCK-8 cell viability assay}

Kupffer cells were seeded in a 96-well plate. After $12 \mathrm{~h}$, cells were exposed to different concentrations of DHA/ AA for $4 \mathrm{~h}$ and then incubated with $10 \mu \mathrm{L} \mathrm{CCK}-8$ (K1018, APExBIO, Houston, USA)) reagent for $2 \mathrm{~h}$. Absorbance was measured at $450 \mathrm{~nm}$ as an indicator of cell activity.

\section{Immunofluorescence}

Kupffer cells were fixed with $4 \%$ paraformaldehyde for $10 \mathrm{~min}$. Each section was soaked with in Tris-buffered saline containing $0.3 \%$ Triton X-100 for $1 \mathrm{~h}$, blocked with $10 \%$ goat serum, and incubated with the primary antibody overnight at $4{ }^{\circ} \mathrm{C}$ and then with the secondary antibody. DAPI was used as a marker for cell nuclei.

\section{SEM observation}

Kupffer cells were prepared and fixed with $2.5 \%$ glutaraldehyde, rinsed with phosphate buffer three times, and dehydrated with gradient ethanol of $50 \%, 70 \%, 80 \%$, and $90 \%$ for 15 min each and then with 100\% ethanol three times. Next, the samples were replaced with t-butanol three times, dried with a freeze-drying apparatus, and then glued to the sample stage. Finally, the samples were plated with a $10-\mathrm{nm}$ gold film with a ion-sputtering apparatus, and the cell morphology was observed under an SEM (Hitachi SU8010, Hitachi Technologies, Tokyo, Japan).

\section{Plasma biochemical measurement}

Plasma ALT, AST, and LDH levels were measured by using an automatic biochemical analyzer (7020, Hitachi, Japan).

\section{H\&E staining}

Fresh livers were fixed with $4 \%$ paraformaldehyde and paraffin-embedded, and the sections were stained using $\mathrm{H} \& \mathrm{E}$. 


\section{PI staining}

Kupffer cells were fixed with $4 \%$ paraformaldehyde for $10 \mathrm{~min}$. The liver tissues were fixed with $4 \%$ paraformaldehyde for $24 \mathrm{~h}$, paraffin-embedded, and sectioned at a thickness of $5 \mathrm{~mm}$. The tissue was then dewaxed in xylene and antigen repair was performed by boiling the sections in the citric acid buffer for $15 \mathrm{~min}$, cooling for $20 \mathrm{~min}$. And then incubating with $4 \mu \mathrm{M}$ PI dye for $30 \mathrm{~min}$ at $37^{\circ} \mathrm{C}$. DAPI was used as a marker for cell nuclei. The Kupffer cells and liver sections were observed using a fluorescent microscope.

\section{Isolation and analysis of cellular plasma membrane fractions}

Ten centimeter dishes of cells were treated with or without $100 \mathrm{ng} / \mathrm{mL}$ LPS for $12 \mathrm{~h}$. Cells were harvested, resuspended in Buffer A (10 mM HEPES, pH 7.4, $1 \mathrm{mM}$ EDTA, $250 \mathrm{mM}$ sucrose) with protease inhibitors, and homogenized with $22 \mathrm{G}$ needles for 30 times. The homogenate was then centrifuged at $800 \mathrm{~g}$ for $10 \mathrm{~min}$ to remove the nuclear pellet. Membrane fractions were prepared by centrifuging at 20,000 $\mathrm{g}$ for $1 \mathrm{~h}$. The supernatant was collected and designated "cytoplasm extracts". The precipitation is membrane fractions and were then solubilized in $100 \mu \mathrm{L}$ Buffer B $(150 \mathrm{mM} \mathrm{NaCl}, 50 \mathrm{mM}$ Tris-Cl pH7.4, 5 mM EGTA, 5 mM EDTA) containing $8 \mathrm{M}$ urea and $2 \%$ SDS. The GPR120 levels in plasma membrane fractions were analyzed by western blot.

\section{Co-immunoprecipitation}

Kupffer cells proteins were extracted, and GPR120 and NLRP3 were immunoprecipitated using protein A/G plus agarose (sc-2003, Santa Cruz, CA, USA) as described previously $^{43}$ The expression of GPR120, NLRP3, and caspase-1 was detected using western blot analysis.

\section{Total RNA isolation and quantitative real-time polymerase} chain reaction

Total RNA was isolated from Kupffer cells or liver tissues using TRIzol reagent (Invitrogen, California, USA) treated with RNase-free DNase, and reverse-transcribed to cDNA using random hexamer primers (Promega). Two microliters of diluted cDNA $(1: 20, v / v)$ was used for realtime PCR with an Mx3000P Real-Time Polymerase Chain Reaction (PCR) System (Stratagene). Glyceraldehyde-3phosphate dehydrogenase (GAPDH) was chosen as a reference gene. All primers were synthesized by Genewiz (Suzhou, China). The primer sequences for qPCR are listed in Supplementary Table S2.

\section{Total protein extraction and western blot analysis}

Total protein was extracted from Kupffer cells or liver tissues as described previously ${ }^{44}$. The protein concentration was determined following the manufacturer's protocols on the BCA Protein Assay kit (Pierce). For 10\% SDS-PAGE, 30 or $50 \mu \mathrm{g}$ proteins were used. Western blot analysis of target proteins was carried out following the protocols provided by the manufacturer. The antibodies used in western blot analysis are listed in Supplementary Table S3. Images were captured using Tannon-5200 (Shanghai, China) and the band density was analyzed using Image $J$ software. For these specific proteins, GAPDH was used as a loading control.

\section{Enzyme-linked immunosorbent assay}

ELISA kits were used to detect the levels of IL-1 $\beta$ and IL-18 (Multisciences (Lianke) Biotech, Co., Ltd, Hangzhou, China) following the manufacturer's protocols.

\section{LC-MS/MS analyses}

The protein solution of immunoprecipitated GPR120 was analyzed by LC-MSMS LC-MS/MS following the manufacturer's protocols.

\section{Statistical analyses}

All statistical analyses were performed using the SPSS 20.0 software for Windows (IBM Corp). All data were expressed as the mean \pm SEM. Statistical analyses were performed using either Student's $t$-test (two-group comparison) or one-way ANOVA analysis of variance (more than two groups) followed by post hoc comparison. For all analyses, the significance level threshold was set at $P<0.05$.

\section{Acknowledgements \\ This study was supported by the National Natural Science Foundation of China} (31772696 and 32072808).

\section{Author contributions}

X.Y. performed study concept and design; X.Y. and G.F. performed development of methodology and writing, review and revision of the paper; Y.L. and J.C. provided acquisition, analysis and interpretation of data, and statistical analysis; Y.Z. provided technical and material support. All authors read and approved the final paper.

\section{Funding}

The authors received no specific funding for this work.

Conflict of interest

The authors declare that they have no conflict of interest.

\section{Ethics statement}

This study was approved by the Animal Ethics Committee of Nanjing Agricultural University, China. Euthanasia and sampling procedures complied with the "Guidelines on Ethical Treatment of Experimental Animals" (2006) No. 398 published by the Ministry of Science and Technology, China, and with the "Regulation Regarding the Management and Treatment of Experimental Animals" (2008) No. 45, published by the Jiangsu Provincial People's Government.

\section{Publisher's note}

Springer Nature remains neutral with regard to jurisdictional claims in published maps and institutional affiliations.

Supplementary Information accompanies this paper at (https://doi.org/ 10.1038/s41419-020-03347-3). 
Received: 1 October 2020 Revised: 6 December 2020 Accepted: 10 December 2020

Published online: 12 January 2021

\section{References}

1. McDonald, B. et al. Programing of an intravascular immune firewall by the gut microbiota protects against pathogen dissemination during infection. Cell Host Microbe 28, 660-668. e664 (2020).

2. Zhang, J. X. et al. Kupffer cell inactivation ameliorates immune liver injury via TNF-alpha/TNFR1 signal pathway in trichloroethylene sensitized mice. Immunopharmacol. Immunotoxicol. 42, 545-555 (2020).

3. Zhai, Y., Petrowsky, H., Hong, J. C., Busuttil, R. W. \& Kupiec-Weglinski, J. W. Ischaemia-reperfusion injury in liver transplantation — from bench to bedside. Nat. Rev. Gastroenterol. Hepatol. 10, 79-89 (2013).

4. Lemasters, J. J. \& Thurman, R. G. Reperfusion injury after liver preservation for transplantation. Annu. Rev. Pharmacol. Toxicol. 37, 327-338 (1997).

5. Chen, X. et al. Pyroptosis is driven by non-selective gasdermin-D pore and its morphology is different from MLKL channel-mediated necroptosis. Cell Res. 26, 1007-1020 (2016).

6. Sborgi, L. et al. GSDMD membrane pore formation constitutes the mechanism of pyroptotic cell death. EMBO J. 35, 1766-1778 (2016).

7. Broz, P. \& Dixit, V. M. Inflammasomes: mechanism of assembly, regulation and signalling. Nat. Rev. Immunol. 16, 407-420 (2016).

8. Hua, S. et al. Glycyrrhizin attenuates hepatic ischemia-reperfusion injury by suppressing HMGB1-dependent GSDMD-mediated kupffer cells pyroptosis. Int. Immunopharmacol. 68, 145-155 (2019).

9. Moradi Sarabi, M. et al. The effects of dietary polyunsaturated fatty acids on miR-126 promoter DNA methylation status and VEGF protein expression in the colorectal cancer cells. Genes Nutr. 13, 32 (2018).

10. Hernando Boigues, J. F. \& Mach, N. The effect of polyunsaturated fatty acids on obesity through epigenetic modifications. Endocrinol. Nutr. 62, 338-349 (2015).

11. Zhang, M. J. \& Spite, M. Resolvins: anti-inflammatory and proresolving mediators derived from omega-3 polyunsaturated fatty acids. Annu. Rev. Nutr. 32 203-227 (2012)

12. Fritsche, K. Fatty acids as modulators of the immune response. Annu. Rev. Nutr. 26, 45-73 (2006).

13. Nakamoto, K. et al. DHA supplementation prevent the progression of NASH via GPR120 signaling. Eur. J. Pharmacol. 820, 31-38 (2018).

14. Hong, L., Zahradka, P., Cordero-Monroy, L., Wright, B. \& Taylor, C. G. Dietary docosahexaenoic acid (DHA) and eicosapentaenoic acid (EPA) operate by different mechanisms to modulate hEPAtic steatosis and hyperinsulemia in fa/ fa Zucker rats. Nutrients 11, 917 (2019).

15. Rutting, S. et al. Dietary omega-6 polyunsaturated fatty acid arachidonic acid increases inflammation, but inhibits ECM protein expression in COPD. Respir. Res. 19, 211 (2018).

16. Ferrucci, L. et al. Relationship of plasma polyunsaturated fatty acids to circulating inflammatory markers. J. Clin. Endocrinol. Metab. 91, 439-446 (2006).

17. Hirasawa, A. et al. Free fatty acids regulate gut incretin glucagon-like peptide1 secretion through GPR120. Nat. Med. 11, 90-94 (2005).

18. Oh, D. Y. et al. GPR120 is an omega-3 fatty acid receptor mediating potent anti-inflammatory and insulin-sensitizing effects. Cell 142, 687-698 (2010).

19. Villegas-Comonfort, S., Takei, Y., Tsujimoto, G., Hirasawa, A. \& Garcia-Sainz, J. A Effects of arachidonic acid on FFA4 receptor: signaling, phosphorylation and internalization. Prostaglandins Leukot. Essent. Fatty Acids 117, 1-10 (2017)

20. Gotoh, C. et al. The regulation of adipogenesis through GPR120. Biochem. Biophys. Res. Commun. 354, 591-597 (2007).

21. Raptis, D. A. et al. GPR120 on Kupffer cells mediates hepatoprotective effects of omega3-fatty acids. J. Hepatol. 60, 625-632 (2014).
22. Oh, D. Y. et al. A Gpr120-selective agonist improves insulin resistance and chronic inflammation in obese mice. Nat. Med. 20, 942-947 (2014)

23. Ichimura, A. et al. Dysfunction of lipid sensor GPR120 leads to obesity in both mouse and human. Nature 483, 350-354 (2012).

24. Sanchez-Reyes, O. B. et al. Free fatty acids and protein kinase $C$ activation induce GPR120 (free fatty acid receptor 4) phosphorylation. Eur. J. Pharmacol. 723, 368-374 (2014).

25. Baffy, G. Kupffer cells in non-alcoholic fatty liver disease: the emerging view. J. Hepatol. 51, 212-223 (2009).

26. Lanthier, N. Targeting Kupffer cells in non-alcoholic fatty liver disease/nonalcoholic steatohepatitis: why and how? World J. Hepatol. 7, 2184-2188 (2015).

27. Chen, J. et al. Kupffer cells in non-alcoholic fatty liver disease: friend or foe? Int J. Biol. Sci. 16, 2367-2378 (2020).

28. Xu, B. et al. Gasdermin D plays a key role as a pyroptosis executor of nonalcoholic steatohepatitis in humans and mice. J. Hepatol. 68, 773-782 (2018).

29. Zhang, K. et al. Silencing IncRNA Lfar1 alleviates the classical activation and pyoptosis of macrophage in hepatic fibrosis. Cell Death Dis. 11, 132 (2020).

30. Chen, $X$. et al. NEK7 interacts with NLRP3 to modulate the pyroptosis in inflammatory bowel disease via NF-kappaB signaling. Cell Death Dis. 10, 906 (2019).

31. Cheng, S. B. et al. Pyroptosis is a critical inflammatory pathway in the placenta from early onset preeclampsia and in human trophoblasts exposed to hypoxia and endoplasmic reticulum stressors. Cell Death Dis. 10, 927 (2019).

32. Chen, N et al. Cathepsin B regulates non-canonical NLRP3 inflammasome pathway by modulating activation of caspase-11 in Kupffer cells. Cell Prolif. 51, e12487 (2018).

33. Li, Z. et al. DHA attenuates hepatic ischemia reperfusion injury by inhibiting pyroptosis and activating PI3K/Akt pathway. Eur. J. Pharmacol. 835, 1-10 (2018).

34. Yang, J. et al. Hemorrhagic shock primes for lung vascular endothelial cell pyroptosis: role in pulmonary inflammation following LPS. Cell Death Dis. 7, e2363 (2016).

35. Liu, Q., Zhang, D., Hu, D., Zhou, X. \& Zhou, Y. The role of mitochondria in NLRP3 inflammasome activation. Mol. Immunol. 103, 115-124 (2018).

36. Slowik, A., Lammerding, L., Zendedel, A., Habib, P. \& Beyer, C. Impact of steroid hormones E2 and P on the NLRP3/ASC/Casp1 axis in primary mouse astroglia and BV-2 cells after in vitro hypoxia. J. Steroid Biochem. Mol. Biol. 183, 18-26 (2018).

37. Shi, J. et al. Cleavage of GSDMD by inflammatory caspases determines pyroptotic cell death. Nature 526, 660-665 (2015).

38. Moniri, N. H. Free-fatty acid receptor-4 (GPR120): cellular and molecular function and its role in metabolic disorders. Biochem. Pharmacol. 110-111, $1-15$ (2016)

39. Mo, X. L., Wei, H. K. Peng, J. \& Tao, Y. X. Free fatty acid receptor GPR120 and pathogenesis of obesity and type 2 diabetes mellitus. Prog. Mol. Biol. Transl. Sci. 114, 251-276 (2013).

40. Bruchard, M. et al. Chemotherapy-triggered cathepsin B release in myeloidderived suppressor cells activates the Nlrp3 inflammasome and promotes tumor growth. Nat. Med. 19, 57-64 (2013).

41. Li, J. et al. Blocking GSDMD processing in innate immune cells but not in hepatocytes protects hepatic ischemia-reperfusion injury. Cell Death Dis. 11, 244 (2020)

42. Li, P. Z., Li, J. Z., Li, M., Gong, J. P. \& He, K. An efficient method to isolate and culture mouse Kupffer cells. Immunol. Lett. 158, 52-56 (2014).

43. Guo, J., Li, Y., Zhao, R. \& Yang, X. Adipokine zinc-alpha2-glycoprotein alleviates lipopolysaccharide-induced inflammatory responses through the beta3-AR PKA/CREB pathway. Cytokine 123, 154742 (2019).

44. Gong, Y. et al. Inactivation of glycogen synthase kinase-3alpha is required for mitochondria-mediated apoptotic germ cell phagocytosis in Sertoli cells. Aging (Albany NY) 10, 3104-3116 (2018). 